OPEN ACCESS

Edited by:

Jie Yu,

People's Liberation Army General

Hospital, China

Reviewed by:

Hyunchul Rhim,

Sungkyunkwan University,

South Korea

Min Woo Lee,

Sungkyunkwan University,

South Korea

${ }^{*}$ Correspondence:

Xiao-yan Xie

xxy1992sys@163.com

†These authors have contributed equally to this work

Specialty section:

This article was submitted to Cancer Imaging and Image-directed

Interventions,

a section of the journal

Frontiers in Oncology

Received: 21 February 2020 Accepted: 08 June 2020

Published: 24 July 2020

Citation:

Huang G, Liu M, Zhang X, Liu B, Xu $M$, Lin M, Kuang $M$, Lu M and

Xie X (2020) Multiple-Electrode

Switching-Based Radiofrequency Ablation vs. Conventional

Radiofrequency Ablation for Single Early-Stage Hepatocellular Carcinoma

Ranging From 2 to $5 \mathrm{Cm}$.

Front. Oncol. 10:1150.

doi: 10.3389/fonc.2020.01150

\section{Multiple-Electrode Switching-Based Radiofrequency Ablation vs. Conventional Radiofrequency Ablation for Single Early-Stage Hepatocellular Carcinoma Ranging From 2 to $5 \mathrm{Cm}$}

Guang-liang Huang ${ }^{1+}$, Ming Liu ${ }^{1+}$, Xiao-er Zhang ${ }^{1}$, Bao-xian Liu ${ }^{1}$, Ming X ${ }^{1}$, Man-xia Lin ${ }^{1}$, Ming Kuang ${ }^{1,2}$, Ming-de $\mathrm{Lu}^{1,2}$ and Xiao-yan Xie ${ }^{1 *}$

${ }^{1}$ Department of Medical Ultrasonics, Institute for Diagnostic and Interventional Ultrasound, The First Affiliated Hospital of Sun Yat-sen University, Guangzhou, China, ${ }^{2}$ Department of Liver Surgery, The First Affiliated Hospital of Sun Yat-sen University, Guangzhou, China

Purpose: To retrospectively compare the treatment outcome of multiple-electrode switching-based radiofrequency ablation (switching RFA) and the conventional RFA for early-stage hepatocellular carcinoma ( $\mathrm{HCC})$.

Methods: A total of 122 patients with single early-stage HCC ranging from 2.1 to $5.0 \mathrm{~cm}$ received ultrasonography-guided percutaneous RFA as the first-line treatment. Seventy-one patients underwent switching RFA, and 51 underwent conventional RFA. Tumor response, major complication, local tumor progression (LTP), and overall survival (OS) were compared between the two groups. Log-rank tests and Cox regression models were used for univariate and multivariate analyses to identify predictors of LTP and OS.

Results: The rate of initial local complete response rates were 100\% $(71 / 71)$ in the switching RFA group and $98.0 \%(50 / 51)$ in the conventional RFA group $(P>0.05)$. No major complication occurred in the switching RFA group, whereas two in the conventional RFA group. After a median follow-up period of 45.9 months (range, 9.8-60.0 months), the rates of LTP in the switching RFA and conventional RFA groups were 19.7\% (14/71) and $41.2 \%(21 / 51)$, respectively. The cumulative LTP rates at 1,3 , and 5 years were 11.3, 20.5, and $20.5 \%$ for switching RFA and $17.6,38.7$, and $46.7 \%$ for conventional RFA, respectively $(p<0.001)$. Switching RFA was an independent factor associated with a lower LTP rate $(p=0.022)$. Five-year OS rates were $75.8 \%$ after switching RFA vs. $66.2 \%$ after conventional RFA ( $p=0.363)$. Extrahepatic recurrence was a significant prognostic factor for OS in multivariable analysis.

Conclusion: Compared with conventional RFA, switching RFA provides a high local tumor control for single early-stage HCC. An ongoing randomized trial might help to clarify the role of this approach for the treatment of HCC.

Keywords: radiofrequency ablation, hepatocellular carcinoma, multiple-electrode switching, local tumor progression, treatment outcome 


\section{INTRODUCTION}

Radiofrequency ablation (RFA) has been widely used in the treatment of early-stage hepatocellular carcinoma (HCC) (14). Compared with surgical resection, the higher local tumor progression (LTP) rate is regarded as a considerable shortcoming of RFA in the treatment of $\operatorname{HCC}(5,6)$. Because of the limited coagulated necrosis induced by RFA, the ability of local control of HCC with RFA greatly depends on tumor size (7-9). To achieve complete ablation of HCC and sufficient safety margin, overlapping ablations are required (10-12). For conventional RFA with one electrode, the electrode is repositioned and reactivated in untreated tumor sites adjacent to the prior ablation zone after each ablation. The hyperechogenicity caused by the early radiofrequency (RF) electrode frequently obscures the tumor boundaries, rendering the reposition of the electrode under ultrasound (US) guidance, which is technically challenging and time consuming even for a relatively small tumor. A multiple-electrode switching system was introduced and enabled to simultaneously use up to three RF electrodes, between which the power is sequentially switched when an impedance spike is encountered, instead of temporarily switching the system off as it occurs in the conventional RF device $(13,14)$. The problem mentioned earlier can be potentially remedied with the introduction of such a multiple-electrode switching system. Several previous reports have shown the encouraging results of multiple-electrode switching-based RFA for the treatment of HCC. However, all these studies were single-arm studies without direct comparison with conventional RFA (15-17). The aim of this study was thus to retrospectively compare the treatment outcome of multiple-electrode switching-based RFA (switching RFA) and the conventional RFA with a single electrode for early-stage HCC.

\section{PATIENTS AND METHODS}

\section{Patients}

From August 2009 to August 2014, a total of 122 patients (105 men, 17 women; mean age $56.3 \pm 12.6$ years, range: $27.0-$ 85.0 years) with HCC were enrolled in this retrospective study (Figure 1). The diagnosis of HCC was based on the noninvasive diagnostic criteria of the American Association for the Study of Liver Diseases (AASLD) or biopsy. The inclusion criteria comprised: (a) adult patients with early-stage HCC and declined resection recommendation; (b) single $\mathrm{HCC}$ with $2.1-5.0 \mathrm{~cm}$ in diameter, treated by conventional cool-tip electrode RFA system or multiple-electrode switching RFA system; (c) liver function status at Child-Pugh class A or B; (d) platelet count over $50 \times$ $10^{9} / \mathrm{l}$; and (e) prothrombin time ratio $>50 \%$. Exclusion criteria include (a) presence of multiple HCCs; (b) presence of vascular invasion or extrahepatic metastases at pre-procedure imaging study; (c) previous treatment for HCC; (d) ongoing anticoagulant treatment that cannot be stopped; and (e) tumor in close proximity to the hepatic hilum. The study was conducted with the approval of the institutional ethics board. Written informed consent was obtained from each patient before treatment.

\section{Radiofrequency Ablation}

US-guided percutaneous RFA was performed under local anesthesia and sedation. Vital signs were continuously monitored during the procedure. Two of the authors (X.Y. X. and M. K., who have 10 and 8 years of experience with RFA, respectively) performed the ablation. RFA was carried out with the Cooltip $^{\mathrm{TM}}$ system (Valleylab, Boulder, CO, USA). For conventional RFA, a 17-gauge internally cooled electrode with a $2-$ to $3-\mathrm{cm}$ long exposed metallic tip was used. Grounding was achieved by attaching a dispersive pad to each of the patient's thighs. Overlapping ablation technique was used, and the generator was set at the maximum power of $200 \mathrm{~W}$ in the impedance automatic mode for 8-12 min. For switching RFA, two or three internally cooled electrodes (Covidien, Boulder, CO) were used, and two grounding pads were placed on each of the patient's thighs. The generator was set at the maximum power of $200 \mathrm{~W}$ in the impedance switching mode for 16-24 min. The selection of electrode number and the length of exposed electrode tip was primarily determined based on tumor size and tumor location. Generally, two RF electrodes were used for tumors 2.1$3.0 \mathrm{~cm}$, and three for tumors $3.1-5.0 \mathrm{~cm}$ in diameter, with an interelectrode distance of $1.0-2.0 \mathrm{~cm}$. Regardless of the ablation with or without a multiple-electrode switching system, the needle track was carefully treated with the electrode being retracted by a $1-\mathrm{cm}$ increment to prevent bleeding and tumor seeding. Contrast-enhanced US was performed immediately after the RFA procedure in order to obtain complete tumor ablation and a 5-mm safety margin as far as possible.

\section{Evaluation of Treatment Response and Follow-Up}

Local efficacy was assessed by a conventional evaluation modality of contrast-enhanced CT or MRI performed 1 month after ablation. According to the CT/MRI results, a response to RFA was classified as complete or incomplete ablation. Complete ablation was defined as non-enhancement in the ablated zone with or without peripheral enhancing rim. Incomplete ablation was indicated when the tissue was still enhanced at the tumor site, and additional ablation was given. If the tumor was still viable after additional ablation, RFA was considered a failure, and the patient was referred for other therapies. The followup protocol included contrast-enhanced US performed at 3 month intervals and contrast-enhanced CT performed every 6 months. LTP was defined as the regrowth of the tumor inside the initially completely ablated nodule. All the ablationrelated complications were classified according to the Society of Interventional Radiology Reporting Standards for image-guided tumor ablation (18). The follow-up duration was defined as the interval between the first RF ablation and either the incidence of the event or the last visit before December 31, 2018. The follow-up for survival analysis was terminated at 60 months. Liver transplantation was censored on the date of surgery.

\section{Statistical Analysis}

Statistical analysis was performed using the SPSS 16.0 software package. A $p$-value of $<0.05$ indicated statistical significance. Continuous data were expressed as the mean \pm standard 


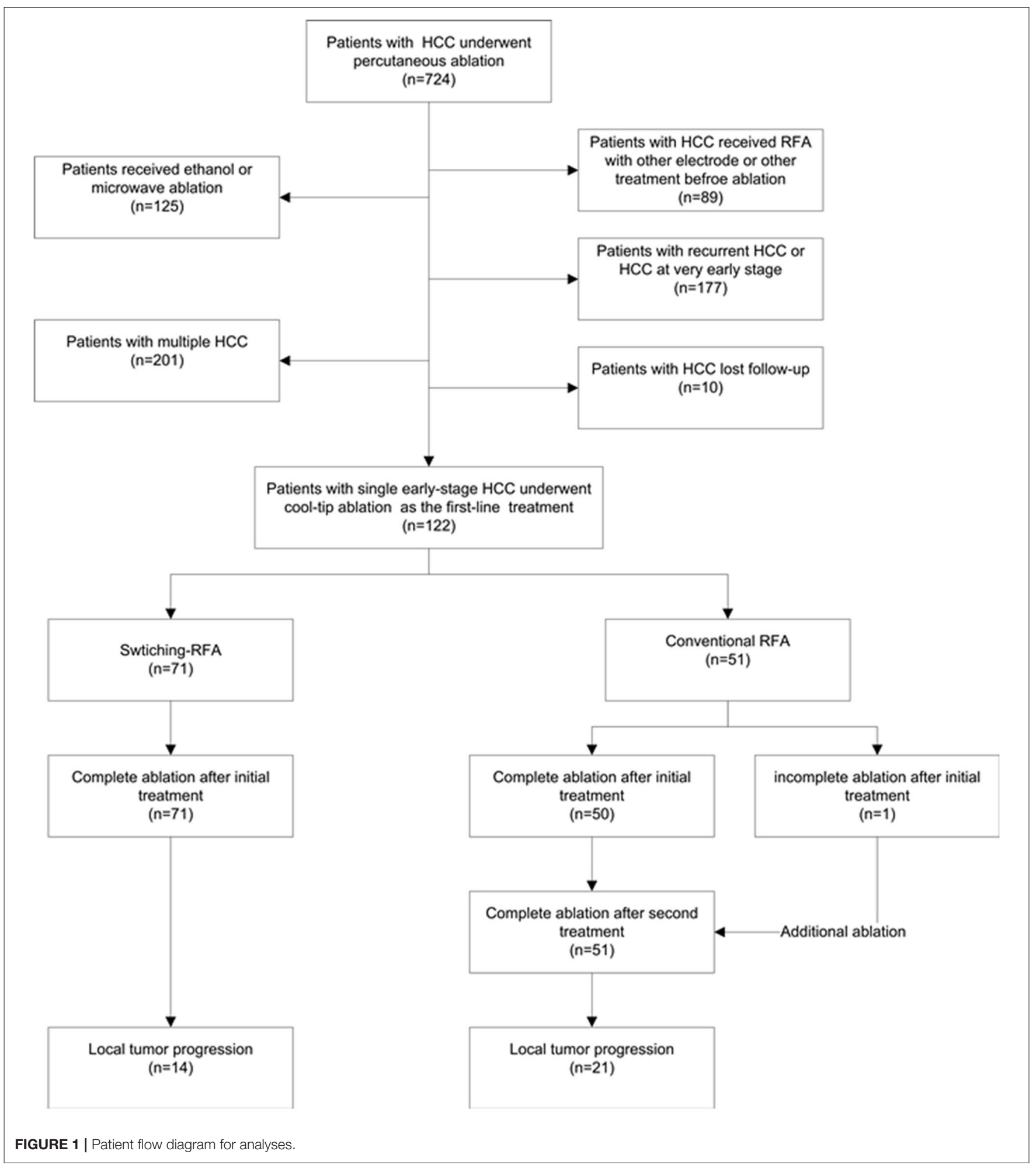

deviation. The chi-squared test or Fisher exact tests were used to compare patients' baseline characteristics. Data on survival were evaluated by the Kaplan-Meier method. The relationship between each of the variables and LTP or overall survival (OS) was estimated by the log-rank test. The variables included age, sex, presence of hepatitis B or C virus infection, Child-Pugh class for liver function, alanine aminotransferase, total bilirubin, albumin, prothrombin time, platelet, serum alpha-fetoprotein, tumor location (perivascular or subcapsular), tumor size, and treatment methods. The variables with a $p$-value of $<0.10$ in the 
log-rank test were introduced in a multivariate Cox proportional hazards model. Perivascular HCC was defined as an index tumor abutting the first- or second-degree branches of a portal or hepatic vein that are $3 \mathrm{~mm}$ or greater in diameter. Subcapsular tumor was defined as an index tumor being $<1.0 \mathrm{~cm}$ from the liver capsule.

\section{RESULTS}

\section{Patients}

Of the 122 eligible patients, 71 (mean age, $55.5 \pm 12.2$ years; range, 27-80 years) underwent switching RFA, whereas 51 (mean age, $57.4 \pm 13.1$ years; range, 27-80 years) underwent conventional RFA. The tumor sizes were $2.8 \pm 0.5 \mathrm{~cm}$ (range, $2.1-4.4 \mathrm{~cm}$ ) in the switching RFA group and $2.8 \pm 0.7 \mathrm{~cm}$ (range, $2.1-4.6 \mathrm{~cm}$ ) in the conventional RFA group. Among 26 perivascular tumors, $18(69.2 \%)$ tumors were abutting portal vein, 6 (23.1\%) abutting hepatic vein, and 2 (7.6\%) abutting inferior vena cava. The baseline clinical characteristics of the two groups are compared in Table 1. No significant differences were

TABLE 1 | Baseline characteristics of patients with single early-stage HCC.

\begin{tabular}{|c|c|c|c|}
\hline Characteristics & $\begin{array}{l}\text { Switching-RFA } \\
\qquad(n=71)\end{array}$ & $\begin{array}{l}\text { Conventional RFA } \\
\qquad(n=51)\end{array}$ & $P$-value \\
\hline Gender (male/female) & $64 / 7$ & $41 / 10$ & 0.125 \\
\hline \multicolumn{4}{|l|}{ Age (y) } \\
\hline$\leq 65$ & 58 & 36 & 0.150 \\
\hline$>65$ & 13 & 15 & \\
\hline Child-Pugh (A/B) & $69 / 2$ & $48 / 3$ & 0.704 \\
\hline Hepatitis B/C $( \pm)$ & $67 / 4$ & $48 / 3$ & 1.000 \\
\hline \multicolumn{4}{|l|}{ Serum ALT level } \\
\hline$\leq 40 \mathrm{U} / \mathrm{L}$ & 46 & 22 & 0.018 \\
\hline$>40 \mathrm{U} / \mathrm{L}$ & 25 & 29 & \\
\hline \multicolumn{4}{|l|}{ Serum total bilirubin } \\
\hline$\leq 34.2 \mathrm{mg} / \mathrm{dl}$ & 65 & 49 & 0.531 \\
\hline$>34.2 \mathrm{mg} / \mathrm{dl}$ & 6 & 2 & \\
\hline \multicolumn{4}{|l|}{ Serum albumin } \\
\hline$\leq 35 \mathrm{~g} / \mathrm{L}$ & 13 & 16 & 0.095 \\
\hline$>35 \mathrm{~g} / \mathrm{L}$ & 58 & 35 & \\
\hline \multicolumn{4}{|l|}{ Prothrombin time } \\
\hline$\leq 14 \mathrm{~s}$ & 64 & 37 & 0.011 \\
\hline$>14 \mathrm{~s}$ & 7 & 14 & \\
\hline \multicolumn{4}{|l|}{ Platelet count } \\
\hline$\leq 100 \times 10^{9} / \mathrm{L}$ & 13 & 20 & 0.010 \\
\hline$>100 \times 10^{9} / \mathrm{L}$ & 58 & 31 & \\
\hline \multicolumn{4}{|l|}{ Serum AFP level } \\
\hline$\leq 200 \mathrm{ng} / \mathrm{ml}$ & 64 & 36 & 0.006 \\
\hline$>200 \mathrm{ng} / \mathrm{ml}$ & 7 & 15 & \\
\hline \multicolumn{4}{|l|}{ Tumor size } \\
\hline $2.1-3.0 \mathrm{~cm}$ & 50 & 35 & 0.832 \\
\hline $3.1-5.0 \mathrm{~cm}$ & 21 & 16 & \\
\hline Perivascular (+/-) & $18 / 53$ & $8 / 43$ & 0.198 \\
\hline Subcapsular (+/-) & $21 / 50$ & $22 / 29$ & 0.122 \\
\hline
\end{tabular}

observed between the two groups in sex, age, Child-Pugh class, presence of hepatitis $\mathrm{B} / \mathrm{C}$ virus infection, serum total bilirubin, serum albumin, tumor size, and tumor location (perivascular or subcapsular tumor), whereas the serum alanine aminotransferase level, prothrombin time, platelet count, and the serum alphafetoprotein level differed significantly.

\section{Tumor Response}

In the switching RFA group, complete ablation was achieved in all tumors in a single session of RFA (Figure 2). In the conventional RFA group, complete ablation was achieved in 50 of 51 tumors in a single session of RFA. One residual tumor reached complete ablation after additional treatment. The rates of initial local complete ablation were $100 \%$ (71/71) in the switching RFA group and $98.0 \%(50 / 51)$ in the conventional RFA group $(P=0.418)$.

\section{Major Complications}

No ablation-related death occurred. No major complication was observed in the switching RFA group, whereas two $(2 / 51,3.9 \%)$ major complications were observed in the conventional RFA group. One patient in the conventional RFA group developed obstructive jaundice as a result of the injury of the bile duct. The patient received percutaneous transhepatic catheter drainage and stent placement. Another patient required US-guided percutaneous gallbladder catheter drainage and antibiotics due to the acute cholecystitis.

\section{Local Tumor Progression}

The overall median follow-up period for all patients was 45.9 months (range, 9.8-60.0 months), with median follow-up periods of 43.0 months (range, 9.8-60.0 months) and 55.3 months (range, 10.3-60.0 months) for switching RFA group and conventional RFA group, respectively $(p=0.107)$. The LTP rate in the switching RFA group was $19.7 \%(14 / 71)$ vs. $41.2 \%(21 / 51)$ in the conventional RFA group $(p=0.010)$. According to the KaplanMeier method, the cumulative LTP rates at 1,3 , and 5 years were $11.3,20.5$, and $20.5 \%$ for switching RFA and 17.6, 38.7, and $46.7 \%$ for conventional RFA, respectively (Figure 3). Risk factors for LTP of HCC were analyzed by the log-rank test, which revealed that the treatment method was significantly associated with LTP $(p=0.018)$. Other factors associated with LTP are described in Table 2. In a multivariable analysis, the treatment method was identified as an independent predictor of LTP (HR $=2.209 ; 95 \%$ CI: $1.123-4.346 ; p=0.022$ ) (Table 3). LTP was treated by repeat RFA $(n=16)$, ethanol ablation $(n=1)$, liver transplantation $(n=1)$, surgical resection $(n=2)$, and TACE $(n=1)$ in the conventional RFA group, whereas repeat RFA $(n=11)$, liver transplantation $(n=1)$, and surgical resection $(n=2)$ in the switching RFA group.

\section{Distant Recurrence}

Intrahepatic distant recurrence had occurred in 25 of 71 (35.2\%) patients in the switching RFA group and in 31 of $51(60.8 \%)$ patients in the conventional RFA group $(p=0.005)$. Extrahepatic recurrence was found in 10 of $71(14.1 \%)$ patients in the switching RFA group and in 11 of $51(21.6 \%)$ patients in the conventional RFA group $(p=0.280)$. 


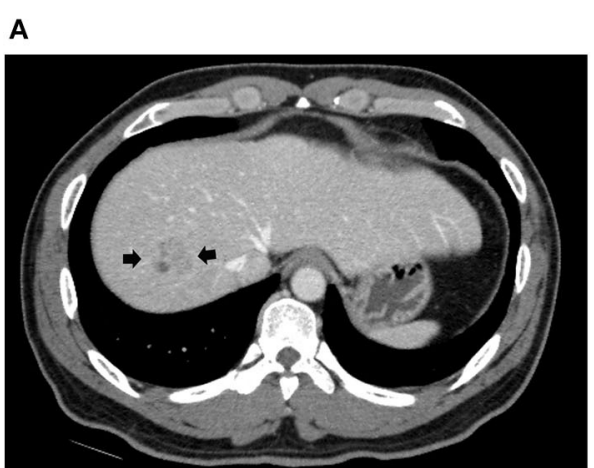

C

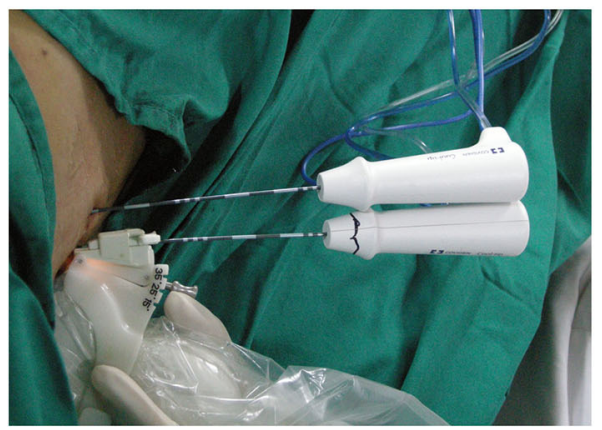

B

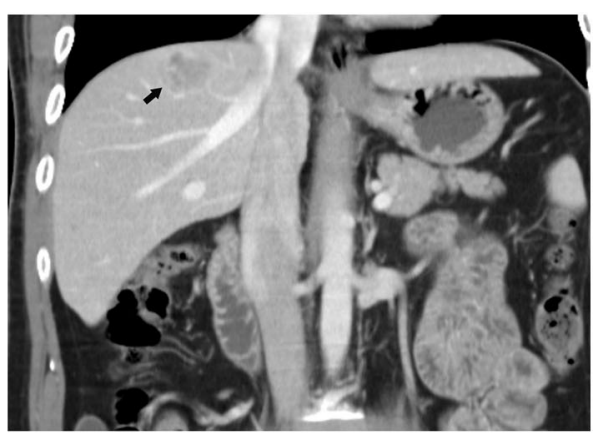

D

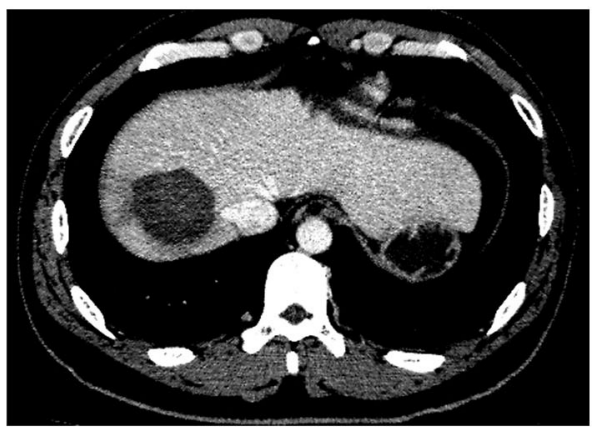

E

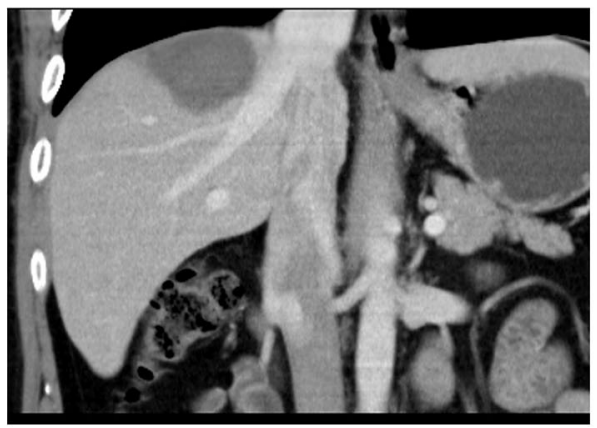

FIGURE 2 | A 39-year-old male patient with HCC who underwent radiofrequency ablation with a multiple-electrode switching system. Pre-ablation CT scans showed a 2.6-cm tumor (arrowhead) in the segment VIII of the liver in the transverse (A) and coronal (B) view. (C) Ultrasound-guided RFA using the multiple-electrode switching system was performed with two RF electrodes for $16 \mathrm{~min}$. CT scans obtained 1 month after ablation showed that the tumor was completely ablated in the transverse (D) and coronal (E) view.

\section{Overall Survival Rates}

Ten $(14.1 \%)$ of 71 patients in the switching RFA group and $13(25.5 \%)$ of 51 patients in the conventional group died during the observation period. The estimated 5-year OS rates were $75.8 \%$ in the switching RFA group and $66.2 \%$ in the conventional RFA group $(p=0.363)$. Of the factors evaluated for association with OS in univariate analysis (Table 4), the following three factors were statistically significant: LTP ( $p=$ $0.004)$, intrahepatic recurrence $(p=0.002)$, and extrahepatic recurrence $(p=0.000)$. Extrahepatic recurrence was found to be a significant risk factor in the Cox proportional hazards regression model (hazard ratio $=15.850$; 95\% CI: 6.169-40.722; $p<0.001$; Table 5).

\section{DISCUSSION}

The present study demonstrated that both switching RFA and conventional RFA achieved satisfactory tumor response for single $\mathrm{HCC}$, ranging from 2.1 to $5.0 \mathrm{~cm}$. Switching RFA appeared to be superior in local control of HCC compared with conventional RFA, whereas similar OS was achieved after both treatments.

Previous studies have reported the LTP rate to be as high as $40 \%$ in patients with early-stage HCC, especially for the intermediate-sized HCC (19). It has been reported that HCC micrometastases may still exist as far as $1 \mathrm{~cm}$ from the main tumor, including the small encapsulated tumors (20). Therefore, at least $0.5 \mathrm{~cm}$ of the ablation margin is required for local 


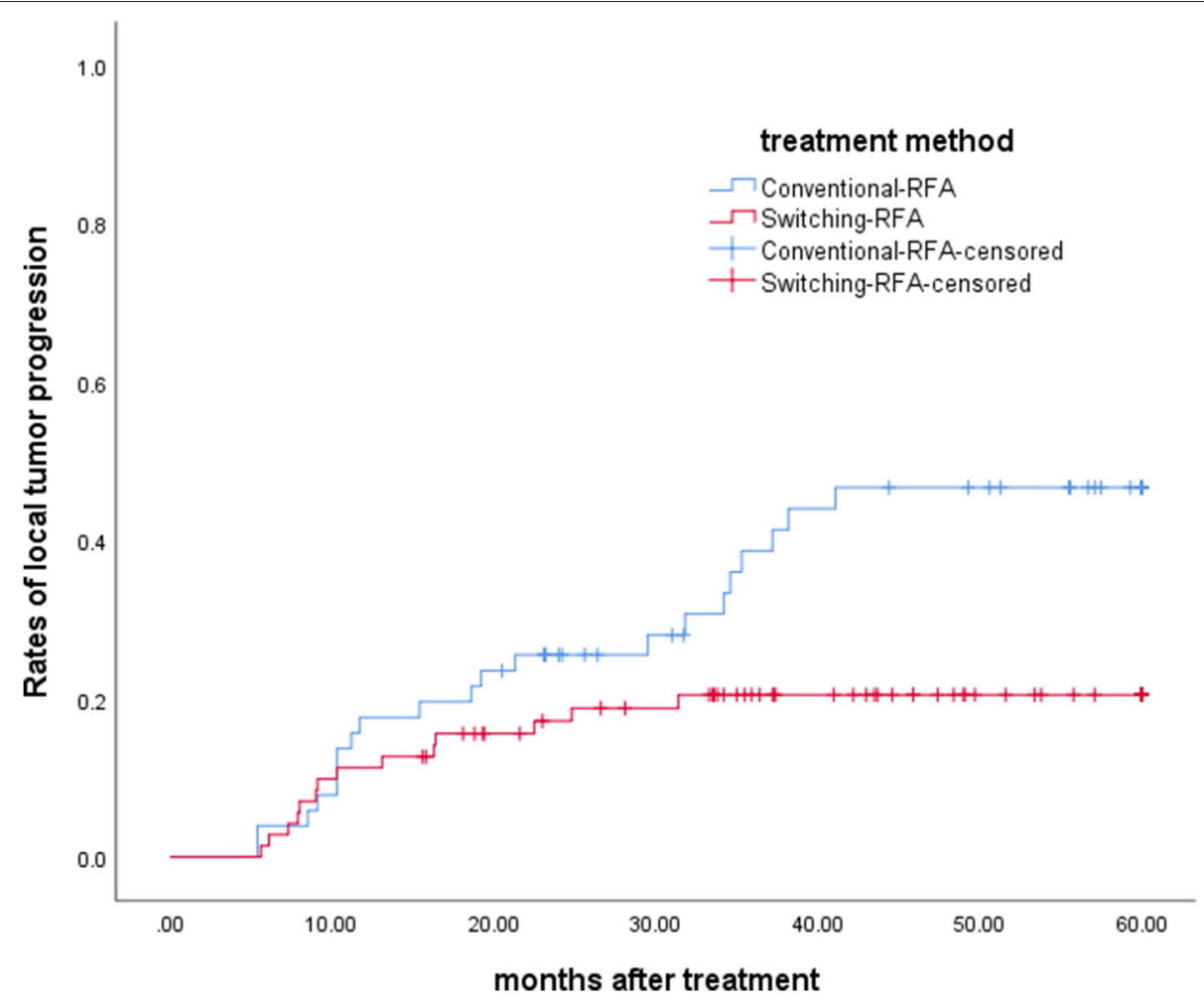

FIGURE 3 | Cumulative LTP survival curves in patients treated with switching RFA or conventional RFA.

TABLE 2 | Univariate analysis of risk factors for local tumor progression of HCC after ablation.

\begin{tabular}{ll}
\hline Variables & Local tumor progression $\boldsymbol{P}$-value \\
\hline Gender (male) & 0.790 \\
Age (>65 years) & 0.601 \\
Hepatitis B/C virus infection (+/-) & 0.382 \\
Child-Pugh class (A/B) & 0.173 \\
Serum ALT level (>40 U/L) & 0.438 \\
Serum total bilirubin (>34.2 mg/dl) & 0.077 \\
Serum albumin (>35 g/L) & 0.089 \\
Platelet count (>100 × 109/L) & 0.773 \\
Prothrombin time (>14 S) & 0.703 \\
Serum AFP level (>200 ng/ml) & 0.962 \\
Tumor size (2.1-3.0/3.1-5.0 cm) & 0.598 \\
Perivascular (+/-) & 0.129 \\
Subcapsular (+/-) & 0.665 \\
Treatment methods (switching/non-switching) & 0.018 \\
\hline
\end{tabular}

+, positive; -, negative; ALT, alanine aminotransferase; AFP, alpha-fetoprotein.

ablative therapy (21-23). However, the commonly used RFA devices show limited ability to create a large ablation zone. To overcome the limitations of conventional RFA, other techniques, including microwave ablation (24), expandable RF electrode (24), and clustered RF electrode (12), have been proposed. In
TABLE 3 | Multivariate analysis of risk factors for local tumor progression of HCC after ablation.

\begin{tabular}{lcc}
\hline Variables & $\boldsymbol{P}$-value & Risk ratio (95\% Cl) \\
\hline Treatment methods & 0.022 & $2.209(1.123-4.346)$ \\
Serum albumin & 0.151 & $\mathrm{NA}$ \\
Serum total bilirubin & 0.096 & $\mathrm{NA}$ \\
\hline
\end{tabular}

NA, not applicable.

theory, Microwave ablation is more efficient than RFA and less influenced by the heat-sink effect. However, the relatively high complication rate limits its clinical use for the tumor close to the critical structures. Expandable electrodes can create a large ablation zone, but it is difficult to see all the time under USguided RFA. Multiple-electrode switching RFA system enabled the creation of significantly larger ablation zones compared with conventional RFA system with a single-electrode in vivo experiment study (14). In the present study, the LTP rates at 1,3 , and 5 years were $11.3,20.6$, and $20.6 \%$ in the switching RFA group, as compared with 17.6, 38.7, and 46.7\% in the conventional RFA group, respectively. Switching RFA provided a better local tumor control than conventional RFA. The result was further supported by the multivariate analysis, which highlighted switching RFA as an independent factor associated with LTP. Previous studies reported that tumor size 
TABLE 4 | Univariate analysis of risk factors for overall survival of HCC after ablation.

\begin{tabular}{ll}
\hline Variables & Overall survival $\boldsymbol{P}$-value \\
\hline Gender (male) & 0.219 \\
Age (>65 years) & 0.334 \\
Hepatitis B/C virus infection(+/-) & 0.481 \\
Child-Pugh class (A/B) & 0.241 \\
Serum ALT level (>40 U/L) & 0.088 \\
Serum total bilirubin (>34.2 umol/L) & 0.151 \\
Serum albumin (>35 g/L) & 0.451 \\
Platelet count (>100 ×109/L) & 0.452 \\
Prothrombin time (>14 s) & 0.689 \\
Serum AFP level $(>200 \mathrm{ng} / \mathrm{ml})$ & 0.197 \\
Tumor size (2.1-3.0/3.1-5.0 cm) & 0.798 \\
Perivascular tumor & 0.158 \\
Subcapsular & 0.114 \\
Treatment methods (switching/non-switching) & 0.363 \\
Local tumor progression & 0.004 \\
Intrahepatic recurrence (+/-) & 0.002 \\
Extrahepatic recurrence (+/-) & 0.000 \\
\hline
\end{tabular}

+, positive; -, negative; ALT, alanine aminotransferase; AFP, alpha-fetoprotein.

TABLE 5 | Multivariate analysis of risk factors for overall survival of HCC after ablation.

\begin{tabular}{lcc}
\hline Variables & $\boldsymbol{P}$-value & Risk ratio (95\% Cl) \\
\hline Extrahepatic recurrence & 0.000 & $15.850(6.169-40.722)$ \\
Local tumor progression & 0.256 & $\mathrm{NA}$ \\
Intrahepatic recurrence & 0.337 & $\mathrm{NA}$ \\
Serum ALT level & 0.532 & $\mathrm{NA}$
\end{tabular}

NA, not applicable.

and tumor location (subcapsular/perivascular location) were two predisposing factors to LTP of $\operatorname{HCC}(3,25,26)$. However, no such correlations were detected in our cohorts.

In the present study, no major complication was observed in the switching RFA group, whereas two major complications occurred in the conventional RFA group. As we mentioned before, the gas induced by the ablation will disturb the reposition of the electrode. Under such circumstances, we had to count the scale marks from both electrode and puncture line and tried to ensure a uniform depth in which a shift of punctate was inevitable, but it cannot be predicted. The likelihood of injuring the neighboring vital structure, such as portal branches, biliary ducts, or gastrointestinal tract, increases. Multiple electrodes in this new system can be inserted into the predetermined location, minimizing the possibility of damaging the important neighboring structure. That could be the reason why no major complication was observed in the switching RFA group in the present study. Although the rate of major complications did not differ between two groups, theoretically, patients may benefit from the multiple-electrode switching-based RFA, and we believe that it can be significant between two groups if large cohorts were analyzed, especially when more large tumors were included.

Interestingly, we found that the LTP rate and the intrahepatic recurrence rate in the switching RFA group were both lower than those in the conventional RFA group; however, no significant differences in OS were observed. Only extrahepatic recurrence was found to be a significant prognostic factor for OS in multivariable analysis. The reason could be explained that most patients with LTP or intrahepatic recurrence were eligible for rescue treatment. However, the estimated overall 5-year survival rate in the switching RFA group was higher than that in the conventional RFA group.

The main limitation of our study is its retrospective nature, which may induce selection bias. Ideally, randomized multicenter controlled clinical trials are needed to provide a complete evaluation of switching RFA for the treatment of HCC. Second, it should be made aware that the outcome of the RFA for HCC is heavily dependent on the expertise of the operators and that we focused only on patients with a single HCC, which may result in differences in the prognostic factors for OS.

In conclusion, our findings from this study demonstrate that the multiple-electrode switching-based RFA is safe and effective in treating single early-stage hepatocellular carcinoma. Compared with conventional RFA, switching RFA provides a high local tumor control for HCC. An ongoing randomized trial might help to clarify the role of this approach for the treatment of HCC.

\section{DATA AVAILABILITY STATEMENT}

All datasets generated for this study are included in the article/supplementary material.

\section{ETHICS STATEMENT}

The studies involving human participants were reviewed and approved by Institutional Review Board approval was obtained from the First Affiliated Hospital of Sun Yat-sen University. The patients/participants provided their written informed consent to participate in this study. Written informed consent was obtained from the individual(s) for the publication of any potentially identifiable images or data included in this article.

\section{AUTHOR CONTRIBUTIONS}

$\mathrm{GH}$ and $\mathrm{XZ}$ accumulated the patients' data and wrote the main manuscript text. BL and MLiu analyzed the results. MX and MLin prepared all figures. MLu and MK conducted the therapy and exam. XX conceived the study, was responsible for the study planning, data analyze and manuscript preparation. All authors wrote and reviewed the main manuscript.

\section{FUNDING}

This work was funded by the National Natural Science Foundation of China, Grant Number: 81501493. 


\section{REFERENCES}

1. Marrero JA, Kulik LM, Sirlin CB, Zhu AX, Finn RS, Abecassis MM, et al. Diagnosis, staging, and management of hepatocellular carcinoma: 2018 practice guidance by the American association for the study of liver diseases. Hepatology. (2018) 68:723-50. doi: 10.1002/hep.29913

2. Lee S, Kang TW, Cha DI, Song KD, Lee MW, Rhim H, et al. Radiofrequency ablation vs. surgery for perivascular hepatocellular carcinoma: propensity score analyses of long-term outcomes. J Hepatol. (2018) 69:70-8. doi: 10.1016/j.jhep.2018.02.026

3. Kutlu OC, Chan JA, Aloia TA, Chun YS, Kaseb AO, Passot G, et al. Comparative effectiveness of first-line radiofrequency ablation versus surgical resection and transplantation for patients with early hepatocellular carcinoma. Cancer. (2017) 123:1817-27. doi: 10.1002/cncr.30531

4. Peng ZW, Zhang YJ, Chen MS, Lin XJ, Liang HH, Shi M. Radiofrequency ablation as first-line treatment for small solitary hepatocellular carcinoma: Long-term results. Eur J Surg Oncol. (2010) 36:1054-60. doi: 10.1016/j.ejso.2010.08.133

5. Hong SN, Lee SY, Choi MS, Lee JH, Koh KC, Paik SW, et al. Comparing the outcomes of radiofrequency ablation and surgery in patients with a single small hepatocellular carcinoma and well-preserved hepatic function. J Clin Gastroenterol. (2005) 39:247-52. doi: 10.1097/01.mcg.0000152746.72149.31

6. Gravante G, Overton J, Sorge R, Bhardwaj N, Metcalfe MS, Lloyd DM, et al. Radiofrequency ablation versus resection for liver tumours: an evidence-based approach to retrospective comparative studies. J Gastrointest Surg. (2011) 15:378-87. doi: 10.1007/s11605-010-1377-6

7. N'Kontchou G, Mahamoudi A, Aout M, Ganne-Carrie N, Grando V, Coderc $\mathrm{E}$, et al. Radiofrequency ablation of hepatocellular carcinoma: long-term results and prognostic factors in 235 Western patients with cirrhosis. Hepatology. (2009) 50:1475-83. doi: 10.1002/hep.23181

8. Lam VW, Ng KK, Chok KS, Cheung TT, Yuen J, Tung H, et al. Risk factors and prognostic factors of local recurrence after radiofrequency ablation of hepatocellular carcinoma. J Am Coll Surg. (2008) 207:209. doi: 10.1016/j.jamcollsurg.2008.01.020

9. Mulier S, Ni Y, Jamart J, Ruers T, Marchal G, Michel L. Local recurrence after hepatic radiofrequency coagulation: multivariate metaanalysis and review of contributing factors. Ann Surg. (2005) 242:15871. doi: 10.1097/01.sla.0000171032.99149.fe

10. de Baere T, Rehim MA, Teriitheau C, Deschamps F, Lapeyre M, Dromain $\mathrm{C}$, et al. Usefulness of guiding needles for radiofrequency ablative treatment of liver tumors. Cardiovasc Intervent Radiol. (2006) 29:6504. doi: 10.1007/s00270-005-0187-9

11. Chen MH, Yang W, Yan K, Zou MW, Solbiati L, Liu JB, et al. Large liver tumors: protocol for radiofrequency ablation and its clinical application in 110 patients-mathematic model, overlapping mode, and electrode placement process. Radiology. (2004) 232:260-71. doi: 10.1148/radiol.2321030821

12. Park MJ, Kim YS, Rhim H, Lim HK, Lee MW, Choi D. A comparison of USguided percutaneous radiofrequency ablation of medium-sized hepatocellular carcinoma with a cluster electrode or a single electrode with a multiple overlapping ablation technique. J Vasc Interv Radiol. (2011) 22:7719. doi: 10.1016/j.jvir.2011.02.005

13. Brace CL, Sampson LA, Hinshaw JL, Sandhu N, Lee FT Jr. Radiofrequency ablation: simultaneous application of multiple electrodes via switching creates larger, more confluent ablations than sequential application in a large animal model. J Vasc Interv Radiol. (2009) 20:118-24. doi: 10.1016/j.jvir.2008.09.021

14. Laeseke PF, Sampson LA, Haemmerich D, Brace CL, Fine JP, Frey TM, et al. Multiple-electrode radiofrequency ablation creates confluent areas of necrosis: in vivo porcine liver results. Radiology. (2006) 241:11624. doi: 10.1148/radiol.2411051271

15. Laeseke PF, Frey TM, Brace CL, Sampson LA, Winter TC 3rd, Ketzler JR, et al. Multiple-electrode radiofrequency ablation of hepatic malignancies: initial clinical experience. AJR Am J Roentgenol. (2007) 188:1485-94. doi: 10.2214/AJR.06.1004

16. Woo S, Lee JM, Yoon JH, Joo I, Kim SH, Lee JY, et al. Small- and mediumsized hepatocellular carcinomas: monopolar radiofrequency ablation with a multiple-electrode switching system-mid-term results. Radiology. (2013) 268:589-600. doi: 10.1148/radiol.13121736

17. Tan Y, Jiang J, Wang Q, Guo S, Ma K, Bie P. Radiofrequency ablation using a multiple-electrode switching system for hepatocellular carcinoma within the Milan criteria: long-term results. Int J Hyperthermia. (2018) 34:298305. doi: $10.1080 / 02656736.2017 .1330495$

18. Ahmed M, Solbiati L, Brace CL, Breen DJ, Callstrom MR, Charboneau $\mathrm{JW}$, et al. Image-guided tumor ablation: standardization of terminology and reporting criteria-a 10-year update. Radiology. (2014) 273:24160. doi: 10.1148/radiol.14132958

19. Morimoto M, Numata K, Kondou M, Nozaki A, Morita S, Tanaka K. Midterm outcomes in patients with intermediate-sized hepatocellular carcinoma: a randomized controlled trial for determining the efficacy of radiofrequency ablation combined with transcatheter arterial chemoembolization. Cancer. (2010) 116:5452-60. doi: 10.1002/cncr.25314

20. Shi M, Zhang CQ, Zhang YQ, Liang XM, Li JQ. Micrometastases of solitary hepatocellular carcinoma and appropriate resection margin. World J Surg. (2004) 28:376-81. doi: 10.1007/s00268-003-7308-x

21. Kim YS, Lee WJ, Rhim H, Lim HK, Choi D, Lee JY. The minimal ablative margin of radiofrequency ablation of hepatocellular carcinoma (> 2 and $<5 \mathrm{~cm}$ ) needed to prevent local tumor progression: 3D quantitative assessment using CT image fusion. AJR Am J Roentgenol. (2010) 195:75865. doi: 10.2214/AJR.09.2954

22. Ye J, Huang G, Zhang $\mathrm{X}, \mathrm{Xu} \mathrm{M}$, Zhou $\mathrm{X}$, Lin $\mathrm{M}$, et al. Threedimensional contrast-enhanced ultrasound fusion imaging predicts local tumor progression by evaluating ablative margin of radiofrequency ablation for hepatocellular carcinoma: a preliminary report. Int J Hyperthermia. (2019) 36:55-64. doi: 10.1080/02656736.2018.1530460

23. Jiang C, Liu B, Chen S, Peng Z, Xie X, Kuang M. Safety margin after radiofrequency ablation of hepatocellular carcinoma: precise assessment with a three-dimensional reconstruction technique using CT imaging. Int J Hyperthermia. (2018) 34:1135-1141. doi: 10.1080/02656736.2017. 1411981

24. Hoffmann R, Rempp H, Erhard L, Blumenstock G, Pereira PL, Claussen $\mathrm{CD}$, et al. Comparison of four microwave ablation devices: an experimental study in ex vivo bovine liver. Radiology. (2013) 268:89-97. doi: 10.1148/radiol.13121127

25. Kim YS, Rhim H, Cho OK, Koh BH, Kim Y. Intrahepatic recurrence after percutaneous radiofrequency ablation of hepatocellular carcinoma: analysis of the pattern and risk factors. Eur J Radiol. (2006) 59:43241. doi: 10.1016/j.ejrad.2006.03.007

26. Lai ZC, Liang JY, Chen LD, Wang Z, Ruan SM, Xie XY, et al. Do hepatocellular carcinomas located in subcapsular space or in proximity to vessels increase the rate of local tumor progression? A meta-analysis. Life Sci. (2018) 207:3815. doi: 10.1016/j.lfs.2018.06.016

Conflict of Interest: The authors declare that the research was conducted in the absence of any commercial or financial relationships that could be construed as a potential conflict of interest.

Copyright $\odot 2020$ Huang, Liu, Zhang, Liu, Xu, Lin, Kuang, Lu and Xie. This is an open-access article distributed under the terms of the Creative Commons Attribution License (CC BY). The use, distribution or reproduction in other forums is permitted, provided the original author(s) and the copyright owner(s) are credited and that the original publication in this journal is cited, in accordance with accepted academic practice. No use, distribution or reproduction is permitted which does not comply with these terms. 